\title{
COMPOSIÇÃO FLORÍSTICA E FITOSSOCIOLOGIA DO CERRADO SENTIDO RESTRITO NO MUNICÍPIO DE ÁGUA BOA - MT
}

\author{
Jeanine Maria Felfili ${ }^{1}$ \\ Paulo Ernane Nogueira ${ }^{1}$ \\ Manoel Cláudio da Silva Júnior ${ }^{1}$ \\ Beatriz Schwantes Marimon ${ }^{2}$ \\ Welington Braz Carvalho Delitti ${ }^{3}$
}

Recebido em 16/06/00. Aceito em 25/09/01.

\begin{abstract}
RESUMO - (Composição florística e fitossociologia do cerrado sentido restrito no município de Água Boa MT).O estudo foi conduzido no município de Água Boa, no Vale do Araguaia, área de ecótono entre o Cerrado e Amazônia, que apresenta um complexo vegetacional com o predomínio do cerrado. O objetivo deste trabalho foi estudar a composição florística e a fitossociologia do cerrado sentido restrito na região. Foram alocadas dez parcelas de 20x50m $\left(1000 \mathrm{~m}^{2}\right)$. Entraram na amostragem todos os indivíduos lenhosos com diâmetro no nível do solo igual ou superior a $5 \mathrm{~cm}$. Além da amostragem com identificação in loco das espécies, foram efetuadas incursões de coleta em áreas próximas, para ampliar o levantamento da composição florística. Foram encontradas 34 famílias botânicas, contendo 60 gêneros e 80 espécies. A família Leguminosae apresentou o maior número de espécies (10), seguida por Myrtaceae (7), Vochysiaceae (6) e Malpighiaceae (5). Outras 19 famílias foram representadas por uma única espécie. As espécies com maior Índice de Valor de Importância foram Curatella americana L., Qualea parviflora Mart., Callisthene fasciculata Mart., Mezilaurus crassiramea (Meiss) Taub. e Byrsonima crassa Nied. Treze espécies estiveram representadas por um único indivíduo. A densidade foi de 995 indivíduos/ha e a área basal de 7,5 $\mathrm{m}^{2}$ /ha. O índice de Shannon encontrado (H') foi de 3,69, evidenciando a alta diversidade da área.
\end{abstract}

Palavras-chave - Amazônia, Brasil, Biodiversidade

\begin{abstract}
Floristic composition and phytosociology of a cerrado sensu stricto in Água Boa - MT). The study was carried out in Água Boa municipality district - MT, in the Araguaia Valley, in a transitional zone between Cerrado and Amazonian. There is a mosaic of vegetation types with the predominance of savannas physiognomies. The aim of this work was to study the floristic composition and the phytosociology of the Cerrado sensu stricto in Água Boa. Ten random plots of 20x50m $\left(1000 \mathrm{~m}^{2}\right)$ were sampled. The minimum diameter for measurement of the woody plants was $5 \mathrm{~cm}$ at the ground level. Besides the sampling with identification in loco of the species, collection incursions were made in the plots and in nearly areas, to enlarge the study of the floristic composition. Thirtyfour botanical families distributed in 60 genera and 80 species were found. The family Leguminosae presented the largest number of species (10), followed by Myrtaceae (7), Vochysiaceae (6) and Malpighiaceae (5). Other 19 families were represented by only one species. The species with higher Index of Importance Value were Curatella americana, Qualea parviflora Mart., Callisthene fasciculata Mart., Mezilaurus crassiramea (Meiss) Taub. and Byrsonima crassa Nied. Thirteen species were represented by only one individual. The density was of 995 plants/ ha and the basal area was of 7,5 $\mathrm{m}^{2} / \mathrm{ha}$. The Shannon index $\left(\mathrm{H}^{\prime}\right)$ was 3,69 evidencing the high diversity of the area.
\end{abstract}

Key words - Savanna, Amazon, Brazil, Biodiversity

\footnotetext{
${ }^{1}$ Universidade de Brasília, Departamento de Engenharia Florestal. CEP. 70910-900. C.P. 04357. e-mail felfili@unb.br.

2 Departamento de Ciências Biológicas, Campus de Nova Xavantina, Universidade do Estado de Mato Grosso.

${ }^{3}$ Universidade de São Paulo, Departamento de Ecologia
} 


\section{Introdução}

O cerrado, apesar de ocupar uma área de quase 2 milhões de $\mathrm{km}^{2}$ e conter uma elevada biodiversidade (Mendonça et al. 1998, Brasil 1999), tem sido pouco valorizado em termos de conservação. Apenas $0,5 \%$ da sua área total está protegida por unidades de conservação de uso restrito (Bruck et al. 1995) e 3,6\% protegidos por alguma categoria de unidade de conservação (Dias 1990). Myers et al. (2000) consideraram o cerrado como um dos 25 ecossistemas do planeta, com alta biodiversidade, que estão ameaçados.

Aproximadamente $37 \%$ da área do cerrado brasileiro já perdeu sua cobertura vegetal primitiva, porém, a Constituição de 1988 não considerou o cerrado como área prioritária para conservação, e as atuais mudanças sugeridas pelo Poder Executivo no Código Florestal, já aprovadas pelo Conselho Nacional do Meio Ambiente (CONAMA), aumentam as possibilidades de sua degradação.

A abertura de extensas áreas para pastagens, lavouras, principalmente de soja, tem contribuído para uma redução drástica das áreas de cerrado.

Levantamentos florísticos e fitossociológicos (Castro 1994; Felfili et al. 1993; 1994; 1997) têm fornecido informações importantes para a compreensão dos padrões biogeográficos do cerrado, e subsidiado a determinação de áreas prioritárias para a conservação.

A região do Complexo Xavantina (Cochrane et al. 1985), onde se situa o município de Água Boa, tem sido objeto de excursões botânicas desde a expedição anglo-brasileira Xavantina-Cachimbo na década de 70 (Askew et al. 1971, Ratter et al. 1973). Posteriormente foram efetuados levantamentos florísticos e fitossociológicos na década de 80, na área do campus avançado da Universidade de Brasília (Felfili et al. 1998), e mais recentemente estes estudos estão se intensificando com o estabele- cimento do campus da Universidade do Estado de Mato Grosso - UNEMAT (Marimon et al. 1998). A região é ampla e possui um mosaico de vegetação contendo formações amazônicas, de cerrado e áreas de transição. $O$ presente trabalho teve como objetivo estudar a composição florística e fitossociológica do cerrado sensu stricto, no Município de Água Boa - MT, no Vale do Rio Araguaia.

\section{Material e Métodos}

O município de Água Boa - MT (13 50'$14^{\circ} 30^{\prime} \mathrm{S}$ e $52^{\circ} 0^{\prime}-52^{\circ} 45^{\prime} \mathrm{W}$ ) pertence à unidade fisiográfica denominada Complexo Xavantina, sistema de terra $\mathrm{n} \mathbf{0} 31$, de acordo com a classificação de Cochrane et al. (1985). A altitude varia de 450 a $500 \mathrm{~m}$ e o clima é do tipo Aw, com temperatura média anual de $24^{\circ} \mathrm{C}$, com máximas mensais de $27,9{ }^{\circ} \mathrm{C}$ e mínimas de 14,7 ${ }^{0} \mathrm{C}$. A precipitação pluviométrica anual está em torno de $1600 \mathrm{~mm}$. A classe de solo predominante na área estudada é Latossolo Vermelho Amarelo (RADAMBRASIL 1981).

A escolha das áreas de amostragem obedeceu aos critérios de cobertura geográfica de pontos extremos e intermediários, dentro dos sistemas de terra propostos por Cochrane et al. (1985), e à disponibilidade de áreas com vegetação natural sob baixo impacto antrópico. Uma adaptação do método Gradsect (Austin \& Heylighers 1990) foi usada para a seleção das parcelas amostrais, que foram distribuídas em uma área de aproximadamente $1.800 \mathrm{~km}^{2}$ (Fig. 1). Foram utilizadas dez parcelas de $20 \mathrm{~m} \times 50 \mathrm{~m}$ $\left(1000 \mathrm{~m}^{2}\right)$, onde foram medidos todos os indivíduos lenhosos com diâmetros no nível do solo igual ou superior a $5 \mathrm{~cm}$.

As parcelas foram alocadas em áreas com o mínimo de alteração antrópica. Além da amostragem com identificação in loco das espécies foram efetuadas incursões de coleta em áreas próximas, para ampliar o levantamento da composição florística. Foram coletadas amos- 


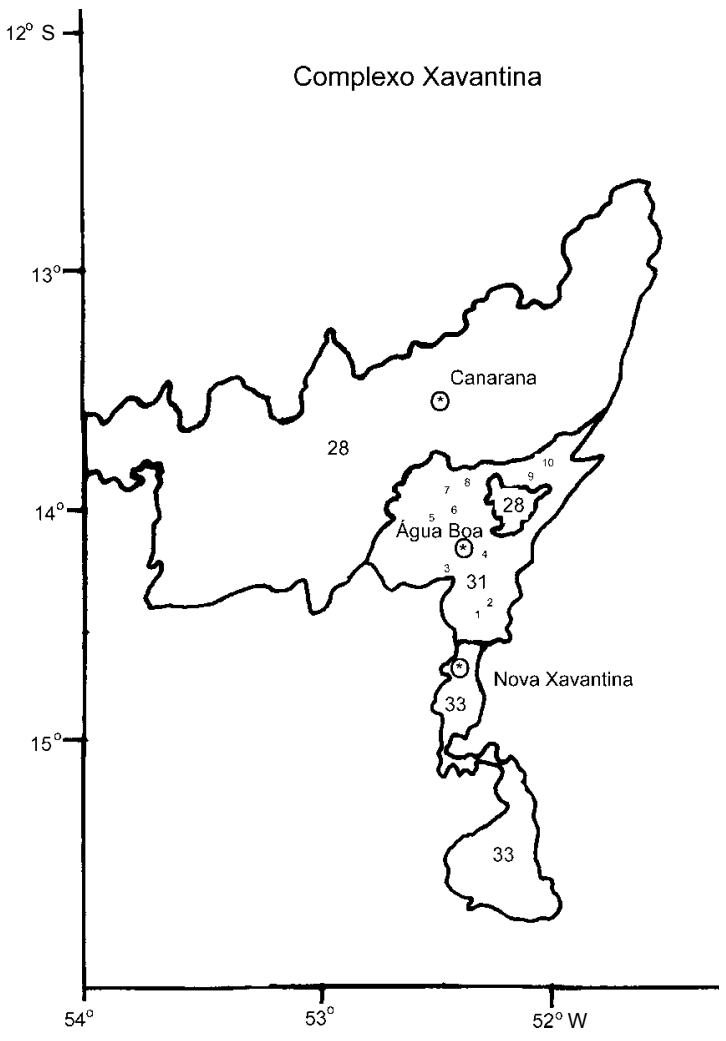

Figura 1. Localização do Município de Água Boa - MT e das parcelas amostrais. 1 a 10 - parcelas amostrais. 28, 31, 33 sistemas de terra conforme Cochrane et al. 1985.

tras botânicas e as exsicatas foram depositadas no herbário da Reserva Ecológica do IBGE. Os nomes botânicos apresentados foram conferidos com a página da WEB do Missouri Botanical Garden (http://mobot.mobot.org/W3T/Search/ vast.html). Os parâmetros fitossociológicos foram calculados de acordo com Curtis \& McIntosh (1951).

\section{Resultados e Discussão}

O sistema de amostragem adotado forneceu indicações de ser apropriado para este estudo, pois o erro padrão da amostragem, tanto para densidade como para a área basal, foi de $9 \%$ da média, indicando que a amostragem foi suficiente para estes parâmetros (ver intervalos de confiança na Tabela 1). Outros estudos realizados com a mesma metodologia em cerrados localizados em um mesmo sistema de terra (Felfili et al.,1993; Felfili e Silva Júnior, 1993, Felfili et al. 1994, 1997) têm indicado que áreas de um hectare, com parcelas dispostas aleatoriamente, são suficientes para a amostragem.

A densidade foi de 995 indivíduos por hectare e a área basal foi de $7,5 \mathrm{~m}^{2} / \mathrm{ha}$. Estes valores estão próximos aos encontrados por Felfili et al. (1997) que, estudando 11 áreas de cerrado nas chapadas Pratinha e dos Veadeiros, encontraram uma média de 1130 árvores por hectare e uma área basal que variou de 5,8 a 11,3 $\mathrm{m}^{2}$ por hectare. Marimon et al. (1998) encontraram no cerrado em Nova Xavantina uma área basal de $13,8 \mathrm{~m}^{2}$ hectare $^{-1}$ e uma densidade de 2980 índivíduos. Ressalte-se que estes autores adotaram um diâmetro mínimo de $3 \mathrm{~cm}$, amostrando, assim, grande número de indivíduos de porte subarbustivo. Na Tabela 1 são apresentados os resultados dos parâmetros fitossociológicos.

Os indivíduos mortos em pé representaram quase $5 \%$ da área basal e do número total de indivíduos, ou a quarta posição no IVI. Outros estudos em cerrado sensu stricto (Felfili \& Silva Júnior 1993; Felfili et al. 1994;1997; Rossi et al. 1998) apresentam variações de 4 a 14\%, sendo que nos ambientes onde o fogo não ocorre com muita freqüência, este valor fica em torno de $5 \%$, o que parece ser o caso do cerrado de Água Boa.

Foram encontradas 34 famílias botânicas contendo 60 gêneros e 80 espécies, sendo que destas, duas não foram determinadas (Lista 1). A família Leguminosae apresentou o maior número de espécies (10), seguido por Myrtaceae (7), Vochysiaceae (6) e Malpighiaceae (5). Outras 19 famílias foram representadas por uma única espécie.

Com relação ao posicionamento das famílias quanto ao IVI, verifica-se que apenas seis delas, Vochysiaceae, Dilleniaceae, Leguminosae, Malpighiaceae, Lauraceae e Myrtaceae, nesta ordem, detêm mais de $50 \%$ deste índice. 
Tabela 1. Fitossociologia do cerrado sentido restrito no município de Água Boa - MT.

\begin{tabular}{|c|c|c|c|c|c|c|c|}
\hline \multirow[t]{2}{*}{ Espécie } & \multicolumn{2}{|c|}{ Densidade } & \multicolumn{2}{|c|}{ Área Basal } & \multicolumn{2}{|c|}{ Frequência } & \multirow[t]{2}{*}{ IVI } \\
\hline & Abs. (ha) & Rel.(\%) & Abs. ( $\left.\mathbf{m}^{2} / \mathbf{h a}\right)$ & Rel.(\%) & Abs (\%) & REL (\%) & \\
\hline Curatella americana & 115,00 & 11,56 & 1,2230 & 16,40 & 90 & 3,88 & 31,84 \\
\hline Qualea parviflora & 92,00 & 9,25 & 0,6471 & 8,68 & 80 & 3,45 & 21,37 \\
\hline Callisthene fasciculata & 68,00 & 6,83 & 0,6707 & 8,99 & 60 & 2,59 & 18,41 \\
\hline Mortas & 48,00 & 4,82 & 0,3625 & 4,86 & 90 & 3,88 & 13,56 \\
\hline Mezilaurus crassiramea & 49,00 & 4,92 & 0,2845 & 3,82 & 20 & 0,86 & 9,60 \\
\hline Byrsonima crassa & 32,00 & 3,22 & 0,1619 & 2,17 & 70 & 3,02 & 8,41 \\
\hline Erythroxylum suberosum & 30,00 & 3,02 & 0,1143 & 1,53 & 80 & 3,45 & 8,00 \\
\hline Terminalia argentea & 25,00 & 2,51 & 0,2762 & 3,70 & 40 & 1,72 & 7,94 \\
\hline Andira paniculata & 22,00 & 2,21 & 0,2190 & 2,94 & 60 & 2,59 & 7,73 \\
\hline Vatairea macrocarpa & 19,00 & 1,91 & 0,1065 & 1,43 & 70 & 3,02 & 6,36 \\
\hline Astronium fraxinifolium & 19,00 & 1,91 & 0,1281 & 1,72 & 60 & 2,59 & 6,21 \\
\hline Magonia pubescens & 16,00 & 1,61 & 0,1429 & 1,92 & 60 & 2,59 & 6,11 \\
\hline Qualea multiflora & 22,00 & 2,21 & 0,1593 & 2,14 & 40 & 1,72 & 6,07 \\
\hline Eugenia dysenterica & 28,00 & 2,81 & 0,2102 & 2,82 & 10 & 0,43 & 6,06 \\
\hline Luehea divaricata & 21,00 & 2,11 & 0,1631 & 2,19 & 30 & 1,29 & 5,59 \\
\hline Salvertia convallariodora & 21,00 & 2,11 & 0,1494 & 2,00 & 30 & 1,29 & 5,41 \\
\hline Sclerolobium aureum & 17,00 & 1,71 & 0,0912 & 1,22 & 50 & 2,16 & 5,09 \\
\hline Qualea grandiflora & 16,00 & 1,61 & 0,1523 & 2,04 & 30 & 1,29 & 4,94 \\
\hline Tabebuia aurea & 10,00 & 1,01 & 0,0918 & 1,23 & 60 & 2,59 & 4,82 \\
\hline Salacia elliptica & 22,00 & 2,21 & 0,1187 & 1,59 & 20 & 0,86 & 4,67 \\
\hline Brosimum gaudichaudii & 18,00 & 1,81 & 0,1121 & 1,50 & 20 & 0,86 & 4,18 \\
\hline Davilla elliptica & 15,00 & 1,51 & 0,0684 & 0,92 & 40 & 1,72 & 4,15 \\
\hline Ouratea spectabilis & 11,00 & 1,11 & 0,0894 & 1,20 & 40 & 1,72 & 4,03 \\
\hline Tabebuia ochracea & 13,00 & 1,31 & 0,0729 & 0,98 & 40 & 1,72 & 4,01 \\
\hline Pseudobombax tomentosum & 12,00 & 1,21 & 0,1092 & 1,47 & 30 & 1,29 & 3,96 \\
\hline Dipteryx alata & 5,00 & 0,50 & 0,1889 & 2,53 & 20 & 0,86 & 3,90 \\
\hline Ouratea hexasperma & 13,00 & 1,31 & 0,0565 & 0,76 & 40 & 1,72 & 3,79 \\
\hline Pseudobombax longiflorum & 10,00 & 1,01 & 0,0695 & 0,93 & 40 & 1,72 & 3,66 \\
\hline Pouteria ramiflora & 6,00 & 0,60 & 0,0916 & 1,23 & 40 & 1,72 & 3,56 \\
\hline Erythroxylum deciduum & 8,00 & 0,80 & 0,0361 & 0,48 & 50 & 2,16 & 3,44 \\
\hline Diospyros burchellii & 14,00 & 1,41 & 0,0491 & 0,66 & 30 & 1,29 & 3,36 \\
\hline Connarus suberosus & 8,00 & 0,80 & 0,0292 & 0,39 & 50 & 2,16 & 3,35 \\
\hline Vochysia rufa & 8,00 & 0,80 & 0,0585 & 0,79 & 40 & 1,72 & 3,31 \\
\hline Lafoensia pacari & 6,00 & 0,60 & 0,0194 & 0,26 & 50 & 2,16 & 3,02 \\
\hline Mouriri pusa & 5,00 & 0,50 & 0,0248 & 0,33 & 50 & 2,16 & 2,99 \\
\hline Myrcia tomentosa & 13,00 & 1,31 & 0,0523 & 0,70 & 20 & 0,86 & 2,87 \\
\hline Hymenaea stigonocarpa & 8,00 & 0,80 & 0,0701 & 0,94 & 20 & 0,86 & 2,61 \\
\hline Bowdichia virgilioides & 6,00 & 0,60 & 0,0748 & 1,00 & 20 & 0,86 & 2,47 \\
\hline Hancornia speciosa & 6,00 & 0,60 & 0,0301 & 0,40 & 30 & 1,29 & 2,30 \\
\hline Aspidosperma tomentosum & 11,00 & 1,10 & 0,0500 & 0,63 & 40 & 0,86 & 3,45 \\
\hline Annona coriacea & 6,00 & 0,60 & 0,0221 & 0,30 & 30 & 1,29 & 2,19 \\
\hline
\end{tabular}


Tabela 1. (continuação).

\begin{tabular}{|c|c|c|c|c|c|c|c|}
\hline \multirow[t]{2}{*}{ Espécie } & \multicolumn{2}{|c|}{ Densidade } & \multicolumn{2}{|c|}{ Área Basal } & \multicolumn{2}{|c|}{ Frequência } & \multirow[t]{2}{*}{ IVI } \\
\hline & Abs. (ha) & Rel.(\%) & Abs. $\left(\mathbf{m}^{2} / \mathbf{h a}\right)$ & Rel.(\%) & Abs (\%) & REL (\%) & \\
\hline Myrcia lanuginosa & 6,00 & 0,60 & 0,0203 & 0,27 & 30 & 1,29 & 2,17 \\
\hline Eriotheca gracilipes & 6,00 & 0,60 & 0,0470 & 0,63 & 20 & 0,86 & 2,10 \\
\hline Dimorphandra mollis & 2,00 & 0,20 & 0,0327 & 0,44 & 20 & 0,86 & 1,50 \\
\hline Piptocarpha rotundifolia & 4,00 & 0,40 & 0,0170 & 0,23 & 20 & 0,86 & 1,49 \\
\hline Myrcia sellowiana & 2,00 & 0,20 & 0,0277 & 0,37 & 20 & 0,86 & 1,43 \\
\hline Casearia sylvestris & 7,00 & 0,70 & 0,0196 & 0,26 & 10 & 0,43 & 1,40 \\
\hline Jacaranda caroba & 3,00 & 0,30 & 0,0168 & 0,23 & 20 & 0,86 & 1,39 \\
\hline Guettarda virburnoides & 3,00 & 0,30 & 0,0163 & 0,22 & 20 & 0,86 & 1,38 \\
\hline Couepia grandiflora & 5,00 & 0,50 & 0,0311 & 0,42 & 10 & 0,43 & 1,35 \\
\hline Acosmium dasycarpum & 2,00 & 0,20 & 0,0151 & 0,20 & 20 & 0,86 & 1,27 \\
\hline Heteropterys byrsonimifolia & 2,00 & 0,20 & 0,0147 & 0,20 & 20 & 0,86 & 1,26 \\
\hline Tabebuia serratifolia & 4,00 & 0,40 & 0,0302 & 0,41 & 10 & 0,43 & 1,24 \\
\hline Bauhinia sp. & 4,00 & 0,40 & 0,0204 & 0,27 & 10 & 0,43 & 1,11 \\
\hline Rourea induta & 4,00 & 0,40 & 0,0166 & 0,22 & 10 & 0,43 & 1,06 \\
\hline Guapira opposita & 2,00 & 0,20 & 0,0199 & 0,27 & 10 & 0,43 & 0,90 \\
\hline Byrsonima basiloba & 2,00 & 0,20 & 0,0161 & 0,22 & 10 & 0,43 & 0,85 \\
\hline Indeterminada & 2,00 & 0,20 & 0,0106 & 0,14 & 10 & 0,43 & 0,78 \\
\hline Machaerium acutifolium & 2,00 & 0,20 & 0,0082 & 0,11 & 10 & 0,43 & 0,74 \\
\hline Rhamnidium elaeocarpum & 2,00 & 0,20 & 0,0076 & 0,10 & 10 & 0,43 & 0,73 \\
\hline Eriotheca pubescens & 2,00 & 0,20 & 0,0065 & 0,09 & 10 & 0,43 & 0,72 \\
\hline Byrsonima coccolobifolia & 1,00 & 0,10 & 0,0132 & 0,18 & 10 & 0,43 & 0,71 \\
\hline Byrsonima verbascifolia & 1,00 & 0,10 & 0,0095 & 0,13 & 10 & 0,43 & 0,66 \\
\hline Hirtella glandulosa & 1,00 & 0,10 & 0,0081 & 0,11 & 10 & 0,43 & 0,64 \\
\hline Neea theifera & 1,00 & 0,10 & 0,0072 & 0,10 & 10 & 0,43 & 0,63 \\
\hline Emmotum nitens & 1,00 & 0,10 & 0,0060 & 0,08 & 10 & 0,43 & 0,61 \\
\hline Myrtaceae & 1,00 & 0,10 & 0,0056 & 0,08 & 10 & 0,43 & 0,61 \\
\hline Tabebuia roseo- alba & 1,00 & 0,10 & 0,0045 & 0,06 & 10 & 0,43 & 0,59 \\
\hline Guapira noxia & 1,00 & 0,10 & 0,0040 & 0,05 & 10 & 0,43 & 0,59 \\
\hline Euplassa inaequalis & 1,00 & 0,10 & 0,0033 & 0,04 & 10 & 0,43 & 0,58 \\
\hline Campomanesia pubescens & 1,00 & 0,10 & 0,0028 & 0,04 & 10 & 0,43 & 0,57 \\
\hline Anacardium occidentale & 1,00 & 0,10 & 0,0025 & 0,03 & 10 & 0,43 & 0,57 \\
\hline Aspidosperma macrocarpon & 1,00 & 0,10 & 0,0022 & 0,03 & 10 & 0,43 & 0,56 \\
\hline Aegiphila lhotskyana & 1,00 & 0,10 & 0,0022 & 0,03 & 10 & 0,43 & 0,56 \\
\hline TOTAIS & 995,00 & 100,00 & 7,4578 & 100,00 & $2320 \quad 1$ & 100,00 & 300,00 \\
\hline
\end{tabular}

Intervalo de Confiança ( $\mathrm{n}^{\circ} / \mathrm{ha}$ ) para densidade $-\mathrm{IC}=\mathrm{P}[995 \pm 17,5]=0,95$ Intervalo de Confiança $\left(\mathrm{m}^{2} / \mathrm{ha}\right)$ para área basal- $\mathrm{IC}=\mathrm{P}[7,5 \pm 0,14]=0,95$ 
Lista 1. Famílias e Espécies da flora lenhosa encontradas no cerrado sentido restrito no Município de Água Boa - MT.

ANACARDIACEAE

Astronium fraxinifolium Schott

Anacardium occidentale L. (=Anacardium curatellifolium St. Hil.)

ANNONACEAE

Annona coriacea Mart.

APOCYNACEAE

Aspidosperma macrocarpon Mart.

Aspidosperma parvifolium A.DC.

Aspidosperma tomentosum Mart.

Hancornia speciosa Gomez var. speciosa

BIGNONIACEAE

Jacaranda caroba (Vell.) A.DC.

Tabebuia aurea (Manso) Benth. \& Hook.f. ex S. Moore

Tabebuia ochracea (Cham.) Standl.

Tabebuia roseo-alba (Ridley) Sandw.

Tabebuia serratifolia (Vahl) Nicholson

BOMBACACEAE

Eriotheca gracilipes (K. Schum.) A. Robyns

Eriotheca pubescens (Mart. \& Zucc.) Schott. \& Endl. ssp. pubescens

Pseudobombax longiflorum (Mart. \& Zucc.) A. Robyns.

Pseudobombax tomentosum (Mart. \& Zucc.) A. Robyns

CELASTRACEAE

Austroplenckia populnea (Reiss.) Lundell

CHRYSOBALANACEAE

Couepia grandiflora (Mart. \& Zucc.) Benth. \& Hook. f.

Hirtella glandulosa Spreng.

COMBRETACEAE

Terminalia argentea Mart. \& Zucc.

COMPOSITAE (ASTERACEAE)

Piptocarpha rotundifolia (Less.) Baker

CONNARACEAE

Connarus suberosus Planch. var. suberosus

Rourea induta Planch. var. induta Baker

DILLENIACEAE

Curatella americana $\mathrm{L}$.

Davilla elliptica St. Hil.

EBENACEAE

Diospyros burchellii Hern.

ERYTHROXYLACEAE

Erythroxylum deciduum St. Hil.

Erythroxylum suberosum St. Hil.

FLACOURTIACEAE

Casearia sylvestris $\mathrm{Sw}$. var. sylvestris 
Lista 1. (continuação).

\section{HIPPOCRATEACEAE}

Salacia elliptica (Mart. ex Schult) G. Don.

ICACINACEAE

Emmotum nitens (Benth.) Miers

LAURACEAE

Mezilaurus crassiramea (Meissn.) Taub.

\section{LEGUMINOSAE}

Acosmium dasycarpum (Vog.) Yakovl. ssp. dasycarpum

Andira paniculata Benth.

Bauhinia sp.

Bowdichia virgilioides $\mathrm{H}$. B. \& K.

Dimorphandra mollis Benth.

Dipteryx alata Vog.

Hymenaea stigonocarpa Mart. ex Hayne

Machaerium acutifolium Vog.

Sclerolobium aureum (Tul.) Benth.

Vatairea macrocarpa (Benth.) Ducke

LYTHRACEAE

Lafoensia pacari St. Hil.

MALPIGHIACEAE

Byrsonima basiloba A. Juss.

Byrsonima coccolobifolia H. B. \& K.

Byrsonima crassa Nied.

Byrsonima verbascifolia (L.) Rich. ex A. L. Juss.

Heteropterys byrsonimifolia A. Juss.

\section{MEMECYLANACEAE}

Mouriri pusa Gardner

MORACEAE

Brosimum gaudichaudii Tréc.

MYRTACEAE

Campomanesia pubescens (DC.) Berg (=Campomanesia bracteolata Kiaersk., Campomanesia corymbosa Blume.)

Eugenia dysenterica Mart. ex DC.

Myrcia lanuginosa DC.

M. tomentosa (Aubl.) DC.

$M$. rorida (Berg) Kiaersk.

M. sellowiana Berg

NYCTAGINACEAE

Guapira noxia (Netto) Lundell

Guapira opposita (Vell.) Reitz

Neea theifera Oerst

OCHNACEAE

Ouratea hexasperma (St. Hil.) Baill. 
Lista 1. (continuação).

Ouratea spectabilis (Mart.) Engl.

PROTEACEAE

Euplassa inaequalis (Pohl) Engl.

Roupala brasiliensis Klotz.

Roupala montana Aubl.

RHAMNACEAE

Rhamnidium elaeocarpum Reiss.

RUBIACEAE

Guettarda viburnoides Cham. \& Schlecht.

SAPINDACEAE

Magonia pubescens St. Hil. (=Magonia glabrata A. St. Hil.)

SAPOTACEAE

Pouteria ramiflora (Mart.) Radlk.

SYMPLOCACEAE

Symplocos rhamnifolia A. DC.

TILIACEAE

Luehea divaricata Mart. \& Zucc.

VERBENACEAE

Aegiphila lhotzkiana L.

VOCHYSIACEAE

Callisthene fasciculata Mart.

Qualea grandiflora Mart.

Qualea multiflora Mart.

Qualea parviflora Mart.

Salvertia convallariaeodora St. Hil.

Vochysiaceae, Leguminosae e Myrtaceae são famílias bem representadas nos cerrados do Brasil Central (Gentry et al. 1997; Mendonça et. al., 1998). A família Lauraceae, uma das mais importantes da Amazônia, porém pouco representada nos cerrados (Gentry 1990), destaca-se em IVI neste estudo, embora representada por uma única espécie. Entretanto, em estudos realizados em savanas amazônicas no Suriname, Guiana e Venezuela, a família Lauraceae alcança valores de IVI bem expressivos (Sarmiento \& Monasterio 1969; Huber, 1982).

O índice de Shannon (H') encontrado foi de 3,69 nats/indivíduo, também na mesma faixa encontrada para as 11 áreas de cerrado estudadas por Felfili et al. (1997), que variou entre 3,11 e 3,56 nats/indivíduo. O índice de equabilidade de Pielou (J') encontrado foi de 0,84. Estes índices evidenciam uma alta diversidade de espécies para a área em estudo. A posição geográfica, pré-amazônica, possivelmente contribuiu para a riqueza e diversidade de espécies verificadas.

O total de espécies encontradas, 80 , corrobora informações de que o padrão de riqueza do componente lenhoso do cerrado sentido restrito apresenta um número inferior a 120 espécies, ocorrendo em diferentes combinações (Oliveira Filho et al. 1989; Felfili et al. 1993; Ratter et al. 1997).

Nove espécies, Tabebuia roseo-alba, Pseudobombax tomentosum, Terminalia argentea, Rhamnidium elaeocarpum, Magonia pubescens, Luehea divaricata, Dipteryx alata, 
Astronium fraxinifolium e Callisthene fasciculata, são indicadoras de solos mesotróficos (Ratter et al. 1996).

As espécies com maior Índice de Valor de Importância foram Curatella americana, Qualea parviflora, Callisthene fasciculata, Mezilaurus crassiramea e Byrsonima crassa. Estas espécies, juntas com Erythroxylum suberosum e Terminalia argentea, detiveram mais de $50 \%$ da área basal total, e quando se acrescentam a este grupo Andira paniculata e Vatairea macrocarpa, verifica-se que estas espécies detêm $50 \%$ do número total de indivíduos, enquanto apenas treze espécies estiveram representadas por um único indivíduo. Esta tendência, onde um grupo pequeno de espécies prevalece sobre as demais, tem sido verificada em outros estudos (Pires et al. 1999, Felfili \& Silva Júnior 1993; Felfili et al. 1994 e 1997; Marimon et. al. 1998, Rossi et al. 1998).

As duas espécies com maior IVI, Curatella americana e Qualea parviflora, apresentam ampla distribuição no domínio dos cerrados. Ratter et al. (1996) analisando a composição florística de 98 áreas de cerrado e das savanas amazônicas, verificaram que essas espécies estiveram presentes, respectivamente, em $71 \% \mathrm{e}$ $60 \%$ das áreas estudadas. Furley et al. (1988) consideraram estas espécies generalistas. Felfili et al. (1993) observam que Curatella americana é mais abundante em cerrados localizados em menores altitudes, o que parece se confirmar no presente trabalho.

Marimon et al. (1998), realizando um levantamento fitossociológico em uma topossequiência de cerrado no município de Nova Xavantina, cerca de $100 \mathrm{~km}$ ao sul do local deste estudo, encontraram como espécies mais importantes Davilla elliptica, Qualea parviflora, Curatella americana e Erythroxylum suberosum, enquanto que Furley et al. (1988), estudando um cerrado $200 \mathrm{~km}$ ao sul desta localidade, encontraram como espécies mais importantes Qualea parviflora, Lafoensia pacarie Curatella ameri- cana, Callisthene fasciculata, a terceira espécie em IVI, é considerada como indicadora de solos ricos em cálcio (Furley et al., 1988; Haridasan \& Araújo, 1988; Ratter et al., 1996), sugerindo que os solos da área em estudo são mesotróficos.

A posição geográfica da área em estudo, pré-amazônica, certamente contribuiu para a elevada riqueza e diversidade de espécies encontradas. Outros estudos com ênfase ecológica na região, tornam-se prementes, para que iniciativas futuras, como recuperação de áreas degradadas ou delimitação de locais estratégicos para a conservação, não sejam totalmente inviabilizadas.

\section{Referências Bibliográficas}

Askew, G.P.; Moffatt, D.J.; Montgomery e R.F. \& Searl, P.L. 1971. Soils and soil moisture as factors influencing the distribution of the vegetation formations of the Serra do Roncador, Mato Grosso. Pp.150-160 In: Mário Guimarães Ferri (Coord.) Anais do III Simpósio sobre o cerrado. E.Bücher/ EDUSP. São Paulo.

Austin, M.P. \& Heyligers, P.C. 1990. New approach to vegetation survey design: Gradsect sampling. Pp. 3151. In: MARGULES, C.R. \& AUSTIN, M.P. Nature Conservation: Cost effective biological surveys and data analysis.

Brasil - Ministério do Meio Ambiente. 1999. Ações prioritárias para a conservação da biodiversidade no Cerrado e Pantanal. Brasília, DF.

Bruck, E.C.; Freire, M.V. \& Lima, M..F. 1995. Unidades de conservação no Brasil, cadastramento e vegetação 1991-1994. Instituto do Meio Ambiente e dos Recursos Naturais Renováveis, Brasília, DF.

Castro, A. A. J. F. 1994. Composição florístico-geográfica (Brasil) e fitossociológica (Piauí-São Paulo) de amostras de cerrado. Tese de doutorado. UNICAMP, Campinas. 520p

Cochrane, T. T.; Sanchez, L.G.; Azevedo, L.G.; Porras, J. A. \& Garver, C.L. 1985. Land in Tropical America. CIAT-EMBRAPA- CPAC. 3. vols. Cali.

Curtis, J.T. \& McIntosh, R.P. 1951. The interrelations of certain analytic and synthetic phytosociological charaters. Ecology 31(3): 476-496.

Dias, B.F.S. 1990. A conservação da natureza.Pp. 583- 
640. In: M. Novaes Pinto, (Org.) Cerrado: caracterização, ocupação e perspectivas. Editora Universidade de Brasília. Brasília, DF.

Felfili, J.M. \& Silva Júnior, M.C. 1993. A comparative study of cerrado (sensu stricto) vegetation in Central Brazil. Journal of Tropical Ecology 9: 277-289.

Felfili, J.M.; Silva Júnior, M.C.; Rezende, A.V.; Machado, B.W.T.; Silva, P.E.N. \& Hay, J.D. 1993. Análise comparativa da florística e fitossociologia da vegetação arbórea do cerrado sensu stricto na Chapada Pratinha, Brasil. Acta botanica Brasilica 6 (2): 27 46.

Felfili, J.M.; Filgueiras, T.S.; Haridassan, M.; Silva Júnior, M.C.; Mendonça, R.C. \& Rezende, A.V. 1994. Projeto biogeografia do bioma cerrado: vegetação e solos. Cadernos de Geociências 12: 75166.

Felfili, J. M.; Silva Junior, M. C.; Rezende, A . V.; Nogueira, P.E.; Walter, B. M. T., Silva, M. A . \& Encinas, J. I. 1997. Comparação florística e fitossociológica do cerrado nas chapadas Pratinha e dos Veadeiros. Pp. 6-11. In: L. Leite \& C.H. Saito (Eds.). Contribuição ao conhecimento ecológico do cerrado. Ed. Universidade de Brasília. Brasília, DF.

Felfili, J.M.; Silva Júnior, M.C. \& Nogueira, P.E. 1998. Levantamento da vegetação arbórea na região de Nova Xavantina, MT. Boletim do Herbário Ezechias Paulo Heringer 3: 63-81.

Furley, P.A.; Ratter, J.A. \& Gifford, d.R. 1988. Observations on the vegetation of eastern Mato Grosso, Brazil. III. The woody vegetation and soils of the Morro de Fumaça, Torixoreu. Pp.191-208 Proceedings of the Royal Society of London B203..

Gentry, A.H. 1990. Floristic similarities and differences between Southern Central America and Upper and Central Amazonia. Pp. 141-157. In: A.H. Gentry (Ed.) Four neotropical rainforests. Yale University Press. London

Gentry, A.H.; Herrera-MacBride,O.; Huber, O.; Nelson,B.W. \& Villamil, C.B. 1997. Regional overview: South America. Pp. 269-307 In: Heywood, V.H. \& Davis, S.D.(coord). Centres of plant diversity. Cambridge, U.K.WWF/IUCN.

Haridasan, M. \& Araújo, G. M. 1988. Aluminiumaccumulating species in two forest communities in the cerrado region of central Brazil. Forest Ecology and Management 24:15-26.

Huber, J. 1982. Significance of savanna vegetation in the Amazon territory of Venezuela. In: G.T. Prance
(Ed.) Biological diversification in the Tropics. Columbia University Press, NY.

Margurran,A. E. 1988. Ecological diversity and its measurement. Chapman \& Hall. London.

Marimon, B.S., Varella, R.F. \& Marimon Júnior, B.H. 1998. Fitossociologia de uma área de cerrado de encosta em Nova Xavantina, Mato Grosso. Boletim do Herbario Ezechias Paulo Heringer 3: 82-101. Mendonça, R.C.; Felfili, J.M.; Walter, B.M.T.; Silva Júnior, M.C.; Rezende, A.V.; Filgueiras, T.S.\& Nogueira, P.E. 1998. Flora vascular do cerrado. Pp. 287- 556. In: M.S.\& S.P. Almeida (Eds.) Cerrado: ambiente e flora. Embrapa- CPAC. Planaltina, DF. Myers, N.; Mittermeier, R.A.; Mittermeier, C.G.; Fonseca, G.A.B. \& Kent, J. 2000 Biodiversity hotspots for conservation priorities. Nature, $\mathbf{4 0 3}$ (6772): 853858.

Oliveira Filho, A.T.; Shepherd, G.D.; Martins, F.R. \& Stubblebine, W.H. 1989. Environmental factors affecting physiognomic and floristic variation in an area of cerrado in central Brazil. Journal of Tropical Ecology 5: 413-431.

Pires, A.; Felfili, J.M. \& Ribeiro, A. A. 1999. Florística e fitossociologia do Cerrado sensu stricto da APA do Cafuringa. Boletim do Herbário Ezechias Paulo Heringer 4:5-20.

Radam-Brasil.1981. Levantamento de Recursos Naturais. Ministério das Minas e Energia 25, Folha SD22 Goiás.

Ratter, J.A.; Richards, P.N.; Argente, G.E. \& Giford, D.R.G. 1973. Observations on the vegetation of northeastern Mato Grosso. Philosophical Transaction fo the Royal Society of London, Series B.; Biological Sciences 226 (880): 449-492.

Ratter, J.A.; Bridgewater, S.; Atkinson, R. \& Ribeiro, J.F. 1996. Analysis of the floristic composition of the brazilian cerrado vegetation II: Comparison of the woody vegetation of 98 areas. Edinburgh Journal of Botany. 53 (2): 153-180.

Ratter, J.A.; Ribeiro, J.F. \& Bridgewater, S. 1997. The brazilian cerrado vegetation and threats to its biodiversity. Annals of Botany 80: 223-230.

Rossi, C.V.; Silva Júnior, M.C.; Santos, C.E.N. 1998. Fitossociologia do estrato arbóreo do cerrado sensu stricto no Parque ecológico Norte, Brasília-DF. Boletim do Herbário. Ezechias Paulo Heringer 2. 49-56.

Sarmiento, G. \& Monaterio, M. 1969. Studies on the savanna vegetation of the Venezuelan Llanos. I. The use of association analysis. Journal of Ecology 57: $579-598$. 